\title{
Review: Genomics of bull fertility
}

\author{
Jeremy F. Taylor ${ }^{1 \dagger}$, Robert D. Schnabel ${ }^{1,2}$ and Peter Sutovsky ${ }^{1,3}$ \\ ${ }^{1}$ Division of Animal Sciences, University of Missouri, Columbia, MO 65211, USA; ${ }^{2}$ Informatics Institute, University of Missouri, Columbia, MO 65211, USA; \\ ${ }^{3}$ Departments of Obstetrics, Gynecology and Women's Health, University of Missouri, Columbia, MO 65211, USA
}

(Received 5 February 2018; Accepted 27 February 2018; First published online 5 April 2018)

\begin{abstract}
Fertility is one of the most economically important traits in both beef and dairy cattle production; however, only female fertility is typically subjected to selection. Male and female fertility have only a small positive genetic correlation which is likely due to the existence of a relatively small number of genetic variants within each breed that cause embryonic and developmental losses. Genomic tools have been developed that allow the identification of lethal recessive loci based upon marker haplotypes. Selection against haplotypes harbouring lethal alleles in conjunction with selection to improve female fertility will result in an improvement in male fertility. Genomic selection has resulted in a two to fourfold increase in the rate of genetic improvement of most dairy traits in US Holstein cattle, including female fertility. Considering the rapidly increasing rate of adoption of high-throughput single nucleotide polymorphism genotyping in both the US dairy and beef industries, genomic selection should be the most effective of all currently available approaches to improve male fertility. However, male fertility phenotypes are not routinely recorded in natural service mating systems and when artificial insemination is used, semen doses may be titrated to lower post-thaw progressively motile sperm numbers for high-merit and high-demand bulls. Standardization of sperm dosages across bull studs for semen distributed from young bulls would allow the capture of sire conception rate phenotypes for young bulls that could be used to generate predictions of genetic merit for male fertility in both males and females. These data would allow genomic selection to be implemented for male fertility in addition to female fertility within the US dairy industry. While the rate of use of artificial insemination is much lower within the US beef industry, the adoption of sexed semen in the dairy industry has allowed dairy herds to select cows from which heifer replacements are produced and cows that are used to produce terminal crossbred bull calves sired by beef breed bulls. Capture of sire conception rate phenotypes in dairy herds utilizing sexed semen will contribute data enabling genomic selection for male fertility in beef cattle breeds. As the commercial sector of the beef industry increasingly adopts fixed-time artificial insemination, sire conception rate phenotypes can be captured to facilitate the development of estimates of genetic merit for male fertility within US beef breeds.
\end{abstract}

Keywords: bull fertility, genomic selection, genome-wide association study, candidate genes, quantitative trait loci

\section{Implications}

Male and female fertility are lowly heritable in cattle, but the reduction in generation interval achieved through the deployment of genomic selection has allowed a rapid improvement of female fertility in US dairy cattle. For several logistical reasons, genetic evaluations are not widely produced for male fertility in either beef or dairy cattle. Because the genetic correlation between male and female fertility is low, little improvement in male fertility is expected as a correlated response to selection for female fertility. To maximize food production from cattle, new approaches must be developed to improve male fertility independently of female fertility.

\footnotetext{
${ }^{\dagger}$ E-mail: taylorjerr@missouri.edu
}

\section{Introduction}

In cattle, fertility is commonly measured using a number of metrics including age at puberty, calving interval, non-return rate, number of services per conception and daughter pregnancy rate (Berry et al., 2014). Perhaps the most useful metric for evaluating fertility in both sexes is the probability of achieving a pregnancy as a result of a single mating to a randomly sampled, but fertile, member of the opposite sex from 4 to $16 \mathrm{~h}$ following the onset of oestrus in the female. Because the majority of beef cattle are multi-sire mated and beef females are exposed to bulls for a period representing two to three oestrous cycles, this latter metric cannot be widely used in the beef industry. However, in the dairy industry, the majority of females are bred by artificial insemination (Al) allowing the outcomes of individual matings to be 
recorded. Despite this, genetic evaluations for fertility in the US dairy industry are calculated only for females. The reason for this is that when service sire is included in the genetic evaluation model, the estimated additive genetic variance for male fertility is 0 (Cole J.B, USDA ARS, personal communication) and this appears to be due to the bull studs' use of service sire conception rate $(\mathrm{SCR})$ data to titrate the number of progressively motile pre-freeze spermatozoa until a uniform non-return rate is achieved, systematically eliminating naturally occurring variation in male fertility (Van Tassell C.P., USDA ARS, personal communication; DeJarnette et al., 2010). As a consequence, selection occurs only for female fertility in both the US beef and dairy industries.

Although the heritabilities of male and female fertility are both generally low (Fortes et al., 2013a; Berry et al., 2014), response to selection is also governed by the extent of phenotypic variation and the length of the generation interval. The advent of genomic selection (Meuwissen et al., 2001) has dramatically reduced generation interval in the US dairy industry (García-Ruiz et al., 2016) and is beginning to similarly impact the US beef industry. This has enabled a remarkable increase in the rate of genetic improvement of production traits and also for female fertility within the US Holstein population, which is shown in Figure 1 (García-Ruiz et al., 2016). While there do not appear to be a large number of studies in cattle, estimates of the genetic correlation between male and female fertility is positive but generally modest in most vertebrate species (from -0.30 to 0.20 for male and female non-return rate in Danish dairy cattle, Hansen, 1979; 'slight' for male and female non-return rate in Norwegian dairy cattle, Syrstad, $1981 ;-0.25,-0.28$ and -0.41 between scrotal circumference and days to calving in Australian Hereford, Angus and Zebu crosses, respectively, Meyer et al., 1991; 0.14 in Manech Tête Rousse sheep, David et al., 2007; 0.15 for male and female contributions to egg fertility in broiler chickens, Wolc et al., 2009; 0.34 for male and female contributions to conception in rabbits, Piles and Tusell, 2012). Consequently, the majority of genes that create variation in

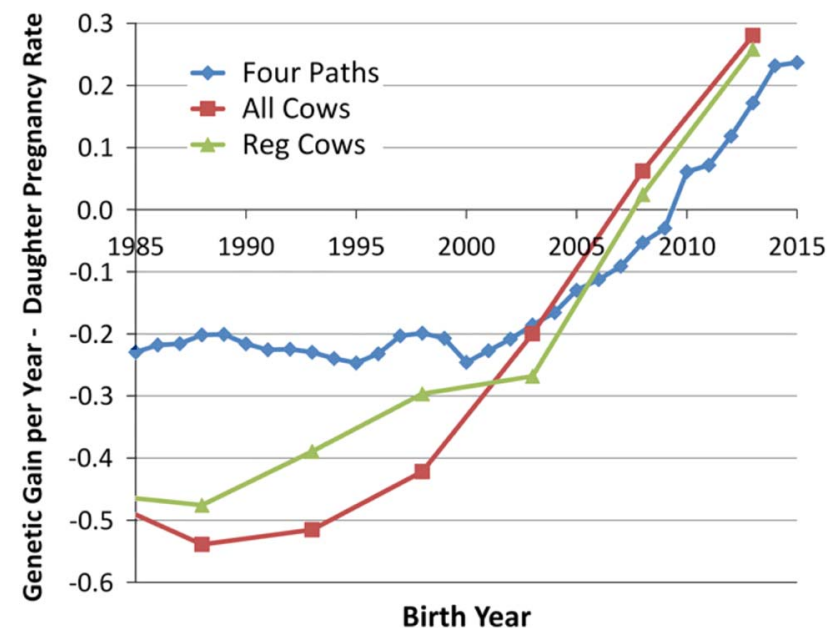

Figure 1 (colour online) Average genetic gain per year for female fertility in US Holsteins (García-Ruiz et al., 2016). male fertility have male-specific functions and selection to improve female fertility will result in a positive, but less than optimal, increase in male fertility. Improvement of the overall efficiency of beef and dairy production will require the ability to identify and eliminate young bulls with sperm abnormalities and unacceptable semen quality (Taylor et al., 2018) and the development of predictors of genetic merit for male fertility that may be applied within and perhaps also across breeds. In this manuscript, we address the current state of knowledge concerning genetic variants responsible for variation in male fertility and the approaches that should be taken to enable the improvement of male fertility in beef and dairy cattle.

\section{Mendelian variants causing variation in male and female fertility}

Of the loci that create genetic variation in both male and female fertility, the most obvious are loss-of-function (LOF) mutations in genes that are essential for life. In the human genome, $7168(33.3 \%)$ of the 21556 annotated genes are essential for life (Chen et al., 2017) meaning that the functionality of at least one copy of each of these genes is required for human life. The number and proportion of essential genes in cattle are probably very similar to those in humans. Mutations which disrupt the functionality of the proteins encoded by essential genes are LOF mutations and for genes located on the autosomes (non-sex chromosomes), homozygosity for a LOF mutation, or heterozygosity of two chromosomes each with a different LOF mutation in the same gene leads to lethality. Because these mutations are transmitted to progeny by both males and females, they are responsible for variation in genetic merit for both male and female fertility. The majority of LOF mutations produce early embryonic loss due to failure to implant or develop. These pregnancy losses are frequently not noticed, but calf losses may also occur in the second and third trimesters of pregnancy or postnatally, manifesting as a genetic defect. These loci are subject to purifying selection because homozygotes are removed every generation leading to relatively small decreases in allele frequency in each successive generation. However, the frequency of some of these LOF lethal alleles can be driven to high levels in a population by the extensive use of $\mathrm{Al}$, which allows carrier bulls to transmit what might otherwise be a rare LOF mutation to a large number of progeny.

Because there is a large number of genes that are essential and these are all targets for mutation, there may be a very large number of lethal LOF mutations within a population. However, the frequency of the majority of these alleles is generally very low and the joint effect of these rare alleles on the mean fertility of the population is small. Because relatively few bulls have been whole genome sequenced (less than 4000 world-wide), the majority of rare variants are yet to be found, either because they were not present in the sequenced animals or because they were detected only once in a sequenced animal and filtered as potentially being a sequence error. Of those that have been discovered, not all 
can confidently be predicted to be a LOF mutation. A mutation that produces a charged amino acid substitution that is predicted to not be tolerated may, or may not, result in the LOF of a protein. Several lethal LOF mutations have been found in cattle by applying the haplotypic insufficiency analytical technique first described by VanRaden et al. (2011). Using this technique, high-density single nucleotide polymorphism (SNP) genotypes such as those produced by the 54001 SNP BovineSNP50 assay (Matukumalli et al., 2009) are first phased so that for each genotyped individual, the two alleles present at each SNP genotype are assigned in a specific order to each of the chromosomes that are present in that individual. Each specific combination of SNP alleles present on a chromosome or chromosomal segment is called a haplotype and the specific pair of haplotypes present within each individual is called a diplotype. Next, the frequencies of haplotypes and diplotypes present in a sample of genotyped animals are tallied for small chromosome segments of, say, 20 consecutive SNPs. The probability of observing no individuals that are homozygous for each haplotype is calculated based on the sample size and the assumption of random inheritance of haplotypes from each parent (Hardy-Weinberg equilibrium). To identify genomic regions that are likely to harbour an autosomal recessive lethal LOF allele, the method of VanRaden et al. (2011) identifies haplotypes that never occur in homozygous form when we would expect to see homozygotes in the sample based on the frequency of the haplotype. The logic behind this method is that if all of the chromosomes in the population that are identified by the same haplotype of 20 SNP alleles harbour a LOF lethal mutation, then every homozygote must be lethal and these individuals will never be seen in the population. Figure 2 provides a schematic representation of this process that shows that when 100000 individuals have been genotyped and the chromosomes have been phased, 20 marker haplotypes that are at a frequency of $2 \%$ in the genotyped sample would be expected to be observed in homozygous form in 40 individuals. When in practice none are observed, the probability that this is due to chance alone is vanishingly small $\left(P_{\mathrm{HWE}}=\left[1-p^{2}\right]^{N}\right.$ $=4 \times 10^{-18}$ ) and we may conclude that the reason that we did not observe any homozygotes is that the haplotype harbours an autosomal recessive lethal LOF allele. Clearly the approach is limited by the need for many genotyped individuals, otherwise low frequency haplotypes will not appear in homozygous form simply due to chance. The approach also assumes that all chromosomes with the same haplotype identified based on alleles at 20 SNPs contain the lethal allele. This may not be the case when the mutation has recently occurred, in which case chromosomes in some individuals will carry the lethal mutation while chromosomes in other individuals will not carry the lethal allele despite the fact that the haplotypes all appear to be identical based upon the marker information. In this case, we will observe fewer homozygotes than expected based on the haplotype frequency, but certainly more than none. This reduces the power of the test and therefore much larger sample sizes are necessary to detect homozygote deficiency when the lethal allele is not perfectly associated with a single haplotype.

A slightly different version of this test was discussed by VanRaden et al. (2011) and implemented by Hoff et al. (2017) that capitalizes on the available pedigree information in genotyped cattle populations. When trios of sire, dam and progeny or patrios of sire, maternal grandsire and progeny have all been genotyped and a particular haplotype is never observed in homozygous form, we can count the number of trios in which the sire and dam are both heterozygotes or the number of patrios where the sire and maternal grandsire are both heterozygotes and calculate the probability of not observing a progeny that is homozygous for the rare haplotype. This is demonstrated in Figure 3 for the case of a patrio where the dam has not been genotyped, the frequency of the rare haplotype in the population is $q$ and the son has a probability of $(2 q+1) / 8$ of being homozygous aa for the rare haplotype ' $a$ ' but is observed to be $A A$ or $A a$. If $N$ such patrios in which the sire and maternal grandsire are both heterozygotes are counted as never producing a homozygous progeny, the probability of this being due to chance alone is $P_{P}=[0.75-0.25 q]^{N}$ and for haplotypes at a frequency of $2 \%$ in the population $P_{P}$ is $1.6 \times 10^{-13}$ for as few as $n=100$ patrios. This may represent many fewer than 300 individuals as sires and maternal grandsires may be common across many patrios. The probability calculation requires only independent assortment of parental alleles in each family

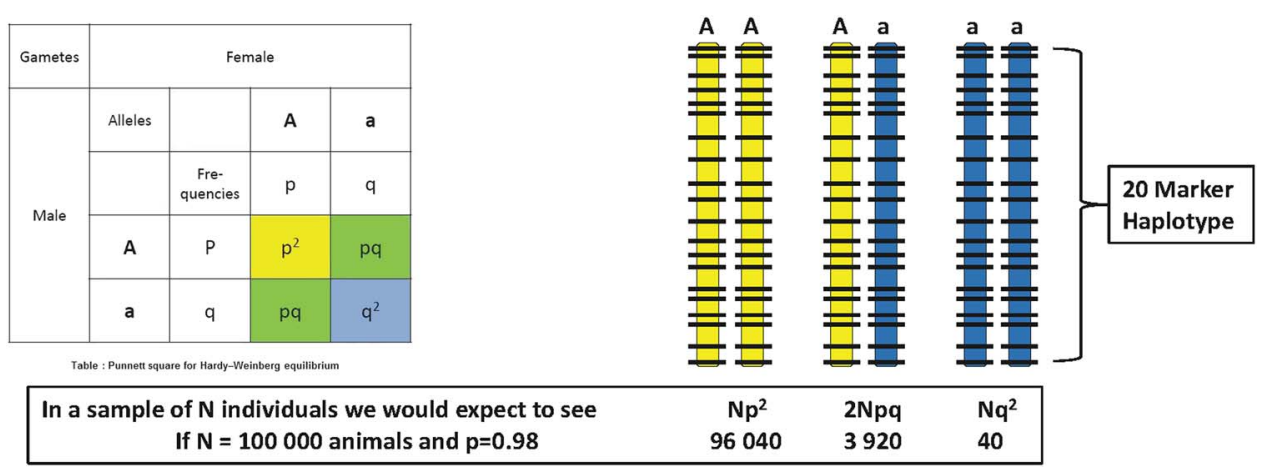

Figure 2 (colour online) Two haplotypes (coloured yellow or blue) defined by alleles present at 20 consecutive single nucleotide polymorphism markers (locations shown as horizontal bars) are present at frequencies of $P=0.98$ and $q=0.02$ in a genotyped sample of size $n=100000$ cattle. On average, 40 animals would be expected to be homozygous for the rare (blue) haplotype. 


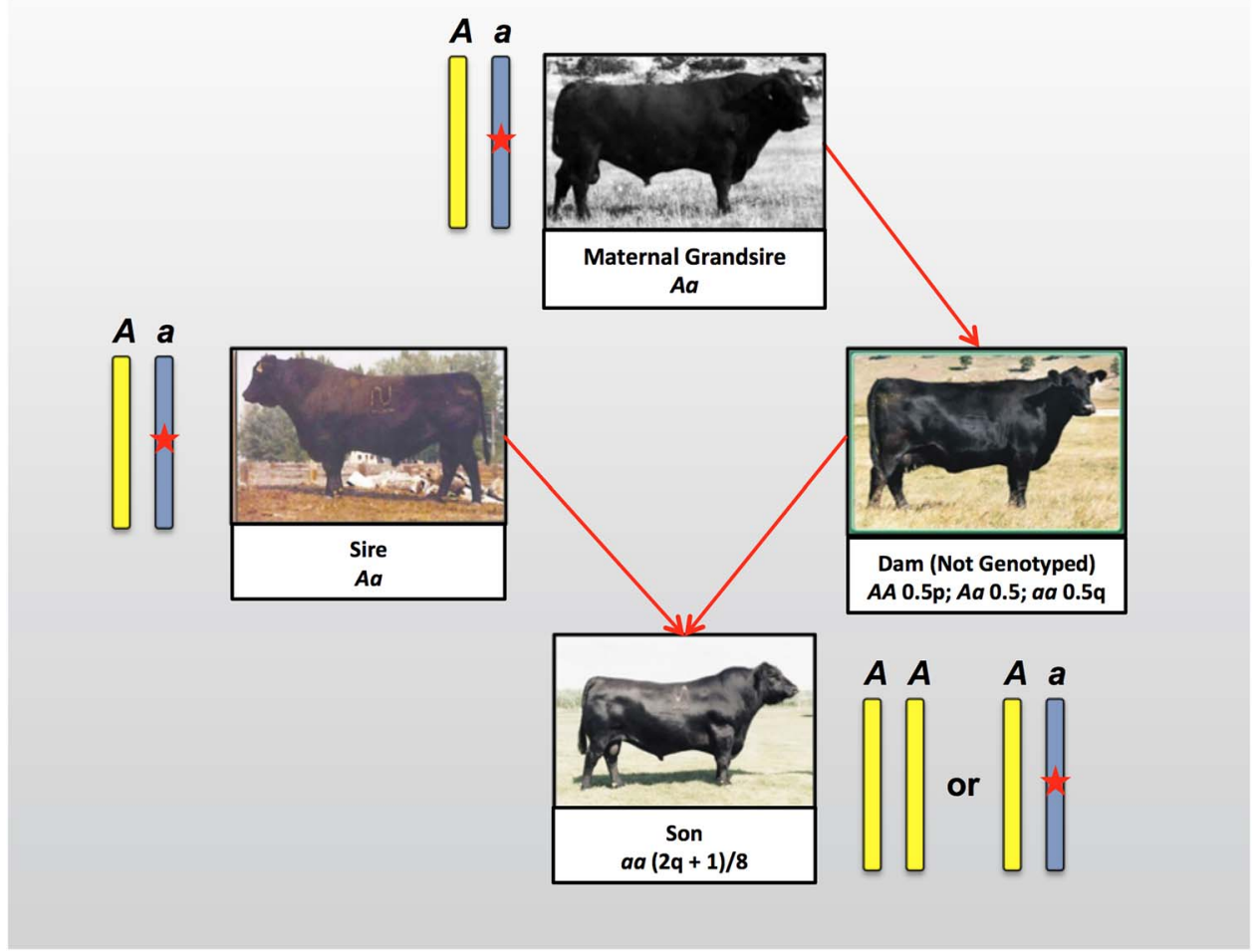

Figure 3 (colour online) An example of a cattle patrio in which the sire and maternal grandsire are both heterozygous for a rare haplotype ' $a$ ' at a frequency $q=1-p$ in the sample and for which no homozygotes are observed. The probability that the son is homozygous for the a haplotype when it does not carry a lethal mutation is $(2 q+1) / 8$.

and not independent families. The approach analyses either overlapping or non-overlapping 20 SNP marker windows sequentially along each chromosome in order to scan the entire genome for the presence of lethal mutations and so there is a multiple testing problem that requires adjustment of the probability values produced for each test to appropriately manage the false discovery rate.

Cole et al. (2017) reported 26 recessive haplotypes that are currently tracked in the US dairy breed genomic evaluation system. Of these, two are fertility related in each of Jersey, Brown Swiss and Ayrshire and eight are fertility related in Holsteins (Table 1). The frequencies of these 12 lethal haplotypes range from $0.37 \%$ to $13 \%$ and average $11.4 \%, 7.2 \%, 1.97 \%$ and $6.7 \%$ in Ayrshire, Brown Swiss, Holstein and Jersey, respectively. The causal mutations responsible for embryonic lethality have been discovered for only nine of the 14 haplotypes. Fritz et al. (2013) performed a genome-wide scan in European dairy breeds for homozygous haplotype deficiency using 47878 Holstein, 16833 Montbeliarde and 11466 Normande animals genotyped with the BovineSNP50 and found 18 haplotypes in Holstein, 11 in Montbeliarde and six in Normande with frequencies ranging from $1.7 \%$ to $9 \%$. Nine of these haplotypes were found to be associated with reductions in fertility when directly tested against conception rate in both heifers and adult cows using heterozygous trio matings, validating the presence of lethal alleles. An additional eight haplotypes were associated with conception rate in heifers or adult cows. Whole genome sequence data from 25 Holstein, 11 Montbeliarde and nine
Normande bulls with important individual contributions to their respective breeds (from $1.1 \%$ to $10.8 \%$ ), sequenced to a depth of coverage from 8.9 to $39.2 \times$ were investigated in an attempt to identify the deleterious mutations associated with eight of the haplotypes associated with fertility in heifers and cows, leading to strong candidates for two lethal mutations in SHBG and SLC37A2 in the Montbeliarde. Six of the recessive lethal haplotypes detected in French Holsteins coincide with those segregating in the US Holstein population (Cole et al., 2017) which is expected considering the broad international use of US bulls. Sahana et al. (2013) identified 17 homozygote deficient haplotypes at frequencies of from $1.4 \%$ to $3.4 \%$ in 7937 Nordic Holsteins genotyped with the BovineSNP50 BeadChip in an analysis in which haplotypes were based on 25 consecutive SNPs. These haplotypes appeared to define eight genomic regions likely to harbour lethal alleles, and of these, six regions were confirmed as having effects on fertility when tested for associations with either non-return rate or calving interval. Remarkably, of all the candidate lethal mutations found in Nordic Holsteins, only the locus on chromosome 21 fully overlaps the locus responsible for Brachyspina in US Holsteins (Cole et al., 2017; Table 1).

Pausch et al. (2015) performed a genome-wide scan for homozygous haplotype deficiency in 25544 Fleckvieh cattle using the Illumina BovineSNP50 BeadChip and found four haplotypes (identified as FH1 through FH4) that were deficient in their observed numbers of homozygotes. Two haplotypes were never observed in homozygous form and 
Taylor, Schnabel and Sutovsky

Table 1 Haplotypes or mutations responsible for embryonic lethality discovered by genome-scanning for haplotype or genotype homozygote insufficiency

\begin{tabular}{|c|c|c|c|c|c|c|}
\hline Breed & Haplotype & $\begin{array}{c}\text { OMIA }^{1} \\
9913 \text { ID }\end{array}$ & Gene $(s)^{2}$ & $\begin{array}{c}\text { Frequency } \\
(\%)\end{array}$ & $\mathrm{BTA}^{3}$ & Region (bp) \\
\hline \multirow[t]{2}{*}{ Ayrshire $^{4}$} & $\mathrm{AH} 1$ & 1934 & UBE3B & 13.00 & 17 & 65921497 \\
\hline & $\mathrm{AH} 2$ & 2134 & RPAP2 & 9.80 & 3 & 51267548 \\
\hline \multirow[t]{2}{*}{ Brown Swiss ${ }^{4}$} & $\mathrm{BH} 1$ & 1825 & - & 6.67 & 7 & 42811272 to 47002161 \\
\hline & $\mathrm{BH} 2$ & 1939 & TUBD1 & 7.78 & 19 & 11063520 \\
\hline \multirow[t]{8}{*}{ Holstein - United States ${ }^{4}$} & $\mathrm{HHO}$ & 151 & FANCI & 2.76 & 21 & 21184869 to 21188198 \\
\hline & $\mathrm{HH} 1$ & 1 & APAF1 & 1.92 & 5 & 63150400 \\
\hline & $\mathrm{HH} 2$ & 1823 & - & 1.66 & 1 & 94860836 to 96553339 \\
\hline & $\mathrm{HH} 3$ & 1824 & SMC2 & 2.95 & 8 & 95410507 \\
\hline & $\mathrm{HH} 4$ & 1826 & GART & 0.37 & 1 & 1277227 \\
\hline & $\mathrm{HH} 5$ & 1941 & TFB1M & 2.22 & 9 & 93223651 to 93370998 \\
\hline & $\mathrm{HHC}$ & 1340 & SLC35A3 & 1.37 & 3 & 43412427 \\
\hline & $\mathrm{HCD}$ & 1965 & $A P O B$ & 2.50 & 11 & 77958995 \\
\hline \multirow[t]{7}{*}{ Holstein - France ${ }^{5}$} & BY & 151 & FANCI & 3.60 & 21 & 20200000 to 22300000 \\
\hline & $\mathrm{HH} 1$ & 1 & APAF1 & 2.60 & 5 & 61400000 to 66200000 \\
\hline & $\mathrm{HH} 2$ & 1823 & - & 1.70 & 1 & 93000000 to 97400000 \\
\hline & $\mathrm{HH} 3$ & 1824 & SMC2 & 2.50 & 8 & 94000000 to 96500000 \\
\hline & $\mathrm{HH} 4$ & 1826 & $G A R T$ & 3.60 & 1 & 1900000 to 3300000 \\
\hline & $\mathrm{HH} 5 / \mathrm{HH} 6$ & 1340 & SLC35A3 & $3.90-4.60$ & 3 & 45800000 to 52600000 \\
\hline & HH13 & 1836 & - & 3.70 & 18 & 56400000 to 58400000 \\
\hline \multirow[t]{6}{*}{ Holstein - Nordic ${ }^{6}$} & $05-1351 / 05-1476$ & 1907 & - & $1.60-2.02$ & 5 & 106713645 to 114405063 \\
\hline & 07-501 & 1909 & - & 1.92 & 7 & 34633456 to 36127497 \\
\hline & $08-1276 / 08-1301 / 08-1326 / 08-1351$ & - & - & $1.48-1.54$ & 8 & 83888935 to 89859523 \\
\hline & $11-926 / 11-976 / 11-1001 / 11-1026$ & 1910 & - & $1.35-1.37$ & 11 & 55345639 to 63759322 \\
\hline & $19-151$ & 1911 & - & 1.95 & 19 & 13154786 to 14478389 \\
\hline & $21-276 / 21-301 / 21-326$ & - & - & $1.94-2.05$ & 21 & 20477690 to 24844501 \\
\hline \multirow[t]{3}{*}{ Holstein - New Zealand ${ }^{7}$} & - & 2036 & TTF1 & 3.52 & 11 & 102485897 to 102515271 \\
\hline & - & 2037 & $R A B G G T B$ & 2.13 & 3 & 69316067 to 69322906 \\
\hline & - & 2038 & RNF20 & 1.82 & 8 & 92911255 to 92935750 \\
\hline \multirow[t]{2}{*}{ Jersey - United States ${ }^{4}$} & JH1 & 1697 & CWC15 & 12.10 & 15 & 15707169 \\
\hline & $\mathrm{JH} 2$ & 1942 & - & 1.30 & 26 & 8812759 to 9414082 \\
\hline Jersey - New Zealand ${ }^{7}$ & - & 2035 & OBFC1 & 6.59 & 26 & 24700354 to 24737868 \\
\hline \multirow[t]{6}{*}{ Montbeliarde ${ }^{5}$} & MH1 & 1827 & $S H B G$ & 9.00 & 19 & 27600000 to 29400000 \\
\hline & $\mathrm{MH} 2$ & 1828 & SLC37A2 & 7.00 & 29 & 27900000 to 29100000 \\
\hline & $\mathrm{MH} 3$ & 1842 & - & 5.10 & 2 & 31500000 to 32800000 \\
\hline & MH5 & 1844 & - & 7.10 & 6 & 73300000 to 74400000 \\
\hline & MH6 & 1845 & - & 2.60 & 7 & 80100000 to 81700000 \\
\hline & $\mathrm{MH8}$ & 1847 & - & 3.50 & 13 & 76400000 to 77600000 \\
\hline \multirow[t]{4}{*}{ Normande $^{5}$} & NH1 & 1851 & - & 1.80 & 24 & 38100000 to 39200000 \\
\hline & $\mathrm{NH} 2$ & 1852 & - & 3.80 & 1 & 145700000 to 146800000 \\
\hline & NH5 & 1829 & - & 1.90 & 7 & 3600000 to 4600000 \\
\hline & NH6 & 1855 & - & 1.90 & 15 & 59800000 to 61100000 \\
\hline Danish Swedish and Finnish Red Cattle ${ }^{8}$ & $\mathrm{~A} 27$ & 1901 & RNASEH $2 B$ & $6.50-16.00$ & 12 & 20101696 to 20755193 \\
\hline Nordic Red Cattle 9 & - & & BTBD9, GLO1, DNAH8 & & 23 & 12291761 to 12817087 \\
\hline \multirow[t]{7}{*}{ Angus $^{10}$} & ANH1 & - & - & 2.30 & 1 & 27786985 to 29095768 \\
\hline & ANH2 & - & - & 7.60 & 4 & 82467969 to 83996686 \\
\hline & ANH3 & - & - & 2.30 & 8 & 62040920 to 63000189 \\
\hline & ANH4 & - & - & 3.20 & 12 & 59989293 to 61258655 \\
\hline & ANH5 & - & - & 3.80 & 15 & 82317986 to 83144172 \\
\hline & ANH6 & - & - & 4.50 & 17 & 46514063 to 47462424 \\
\hline & ANH7 & - & - & 4.40 & 29 & 43043207 to 44243444 \\
\hline \multirow[t]{4}{*}{ Fleckvieh $^{11}$} & $\mathrm{FH} 1$ & 1957 & - & 2.90 & 1 & 1668494 to 6187555 \\
\hline & $\mathrm{FH} 2$ & 1958 & $S L C 2 A 2$ & 4.10 & 1 & 97239973 \\
\hline & $\mathrm{FH} 3$ & 1959 & - & 3.30 & 10 & 26929817 to 35479280 \\
\hline & $\mathrm{FH} 4$ & 1960 & SUGT1 & 3.30 & 12 & 11131497 \\
\hline \multirow[t]{5}{*}{ Belgian Blue ${ }^{7}$} & - & 2042 & EXOSC4 & 1.33 & 14 & 1947198 to 1949074 \\
\hline & - & 2043 & MED22 & 1.15 & 11 & 104305076 to 104311650 \\
\hline & - & 2039 & MYH6 & 4.99 & 10 & 21325414 to 21344965 \\
\hline & - & 2041 & RPIA & 1.89 & 11 & 47220160 to 47254704 \\
\hline & - & 2040 & SNAPC4 & 5.13 & 11 & 103884749 to 103905548 \\
\hline
\end{tabular}

${ }^{1}$ Online Mendelian inheritance in animals. Taxon ID 9913 represent cattle.

${ }^{2}$ Multiple listed genes represent a deletion.

${ }^{3}$ Bos taurus chromosome.

${ }^{4}$ Cole et al. (2017)

${ }^{5}$ Fritz et al. (2013).

${ }^{6}$ Sahana et al. (2013).

${ }^{7}$ Charlier et al. (2016).

${ }^{8}$ Kadri et al. (2014)

${ }^{9}$ Sahana et al. (2016).

${ }^{10} \mathrm{Hoff}$ et al. (2017). Haplotypes not validated as fertility associated.

${ }^{11}$ Pausch et al. (2015). 
the frequencies of all four haplotypes ranged from $2.9 \%$ to $4.1 \%$. Insemination success was reduced by $6.64 \%$ and $5.99 \%$ in $\mathrm{FH} 1$ and $\mathrm{FH} 4$ carrier-to-carrier matings, respectively. A 4.06\% decline in insemination success and a 4.3\% reduced first-year survival rate of progeny was observed for FH3 carrier-to-carrier matings. Insemination success and stillbirth rate were not affected in $\mathrm{FH} 2$ carrier-to-carrier matings; however, juvenile mortality in progeny was increased by $6.6 \%$ compared with the survival of progeny from non-risk matings. Using whole genome sequence data from 263 animals from ten different cattle breeds (including 145 Fleckvieh and 15 Simmental) with an average of $10 \times$ sequence coverage, these authors identified strong functional candidate mutations underlying two of the haplotypes. A small indel producing a frameshift in $S L C 2 A 2$ was shown to activate cryptic splice sites in the processed mRNA leading to aberrant splicing at exon 7, while a missense mutation in SUGT1 was predicted to be highly damaging to SGT1 protein function. With many fewer genotyped animals, Hoff et al. (2017) analysed BovineSNP50 data for 3961 registered Angus animals and identified seven haplotypes genome-wide that were predicted to harbour autosomal recessive lethal alleles. These were not validated to directly affect fertility or survival rates but ranged in frequency from $2.3 \%$ to $7.6 \%$. Despite an analysis of sequence data from 109 bulls resequenced to an average $27 \times$ depth of coverage from which 1 to 27 bulls (average of 11.4 bulls were sequenced for each haplotype) were predicted to be carriers of each recessive lethal haplotype, no strong candidates for any of the lethal mutations were found despite six of the regions being detected as harbouring from 1 to 118 concordant (never homozygous) mutations. Because Hoff et al. (2017) restricted their attention to SNPs, many of the causal variants may be small or large insertions or deletions that are not easily or reliably detected from variant calling pipelines.

Advantages of the haplotype-based analysis approach are that it does not require any reproductive data and can be accomplished as a by-product of genotyping animals to enable genomic selection. As very large numbers of animals are genotyped within each breed (over 2 million Holsteins https://www.cdcb.us/Genotype/cur_density.html and over 300000 registered Angus https://www.angus.org/pub/news room/releases/062717-single-step.html animals have now been genotyped in the United States) it becomes possible to detect haplotypes containing autosomal recessive lethal mutations that are at very low frequency within a population. For example, Holstein Haplotype $4(\mathrm{HH} 4)$ is at a frequency of only $0.37 \%$ in the US Holstein population (Cole et al., 2017). The disadvantage of the approach is that haplotype-based selection is possible only when all chromosomes possessing the haplotype signature harbour the lethal allele leading to the complete absence of homozygotes for the haplotype. For recent mutations, where the lethal allele exists only on some of the chromosomes possessing the haplotype that has a homozygous deficiency, additional family analyses must be performed to identify which families transmit the lethal version of the haplotype and which transmit the viable haplotype. The problem is that the analysis does not actually identify which mutation within the genomic region spanned by the haplotype is the cause of the lethal phenotype. To accomplish this requires sequencing individuals that carry the lethal haplotype to identify candidate mutations that can then be directly tested by genotyping within the population for the absence of homozygotes. Fritz et al. (2013) warn that the existence of strong linkage disequilibrium may lead to the causative variants actually being located outside of the intervals defined by the haplotypes. This appears to be supported by the results in Table 1 which summarize studies performed in Holstein cattle world-wide and reveal that lethal haplotypes detected in different Holstein strains may not completely overlap, although we presume that the same causal variant was detected.

Mesbah-Uddin et al. (2018) utilized $10 \times$ average depth of coverage whole genome sequence data produced for 67 Holsteins, 27 Jerseys and 81 Nordic Red Cattle to identify 8480 large deletions ( $199 \mathrm{bp}$ to $773 \mathrm{~kb}$; mean $4.5 \mathrm{~kb}$; median $1 \mathrm{~kb})$. The deletions were validated to have an overall false discovery rate of $8.8 \%$ using Illumina BovineHD genotype intensity data produced for 26 of the sequenced Holsteins, chromosome breakpoint assembly and alignment and the sequencing of PCR amplicons spanning breakpoints. By examining the deletion genotypes of the sequenced individuals, the authors found 5000 deletions for which at least one sequenced individual was homozygous. Among these were 167 deleted genes that were demonstrated to be non-essential based on the occurrence of live homozygote individuals. This study is essentially the reciprocal of the haplotype-based approaches just discussed as it can unequivocally identify genes to be non-essential via the existence of living animals that are homozygous knockouts. While the resolution at which candidate lethal mutations are scanned is dramatically increased by considering whole genome sequence variation, the identification of putative lethal mutations is limited by the relatively small sample of sequenced individuals. However, a $\sim 525 \mathrm{~kb}$ deletion on chromosome 23, which is known to cause stillbirth in Nordic Red Cattle (Sahana et al., 2016) was among the haplotypes that were not found in homozygous form in the sequenced animals.

Kadri et al. (2014) performed a genome-wide association study (GWAS) in 10099 bulls from five European dairy breeds using estimated breeding values of the bulls for an index of cow fertility traits and found 14 quantitative trait loci (QTLs) at genome-wide significance, of which the most significant was located on chromosome 12 . By repeating the analysis within each of the breeds, they identified that this QTL was primarily segregating in Finnish Ayrshire and Swedish Red, but was not detectable in Holstein-Friesian, Danish Red or Jerseys. A haplotype-based analysis identified a single haplotype (A27) that was found to contain a $660 \mathrm{~kb}$ deletion that contained four genes, that produced an increase in milk production in heterozygous animals but that was an embryonic lethal in homozygotes likely due to the 
loss of RNASEH2B. The frequency of this deletion haplotype was $6.5 \%, 11.5 \%$ and $16 \%$ in Danish, Swedish and Finnish Red Cattle, respectively.

Charlier et al. (2016) resequenced the genomes of 496 Holstein-Friesian $\times$ Jersey animals and 50 Belgian Blue Cattle to an average depth of $11 \times$ and resequenced the exomes of 78 animals from six Bos taurus breeds to an average depth of $40 \times$. A total of 186112 exonic variants, including 1377 stop-gain, 112 stop-loss, 3139 frameshift, 1341 splice-site, 85338 missense, and 92163 synonymous variants were discovered. Of the missense variants, 22939 were predicted by SIFT and/or PolyPhen to be disruptive or damaging to protein function. From these, 3779 candidate variants (frameshift, splice-site, stop-gain and missense variants predicted to be damaging and/or deleterious) for embryonic loss were genotyped in 35000 New Zealand dairy cattle (296 LOF and 3483 missense) and 1050 were genotyped in 6300 Belgian Blue cattle (108 LOF, 942 missense). From the produced genotype data, the authors estimated that $15.5 \%$ of the tested LOF variants and $5.9 \%$ of the tested missense variants were embryonic lethal mutations. Nine common LOF variants were confirmed to be embryonic lethal mutations based upon the absence of homozygotes in carrier $\times$ carrier matings (Table 1 ).

Table 1 contains a list of haplotypes or mutations recently discovered via genome scans for homozygote insufficiency that have been demonstrated to affect fertility in several beef and dairy breeds. There are several remarkable observations from the data in this table. First, the average frequency of lethal mutations detected in cattle populations to date is about 3.8\%, which is much larger than you would expect for loci subjected to strong purifying selection. This is clearly due to the extensive use of $\mathrm{Al}$ in these populations, which results in the strong enrichment of alleles present within the genomes of selected bulls that go on to have large numbers of progeny. However, the number of lethal loci detected to date in numerically large breeds such as Holstein (17) is far greater than for the numerically smaller breeds such as Jersey (3), Brown Swiss (2) and Ayrshire (2). Furthermore, the average frequency of lethal alleles in Holsteins $(2.4 \%)$ is considerably lower than in Jersey (6.66\%), Brown Swiss (7.23\%) and Ayrshire (11.4\%). This likely reflects a greater number of genotyped animals used to detect a larger number of rarer alleles, but it also may suggest that the larger number of bulls used in $\mathrm{Al}$ in numerically large breeds allows a greater enrichment of lethal loci in the breed, albeit at lower individual frequencies. While it is difficult to make conclusions about the identities of mutations based on haplotype data (Charlier et al. (2016) found three lethal mutations located within a $1.82 \mathrm{Mb}$ region of chromosome 11 in two breeds) the data in Table 1 suggest that the majority of the common lethal alleles are breed specific. That is, these mutations occurred after breed formation and were driven to relatively high frequencies within the respective breeds by the use of selective breeding. On the other hand, there is also evidence for potentially different lethal loci segregating within subpopulations of Holsteins. Despite the widespread use of high merit US Holstein bulls world-wide, the loci found by Sahana et al. (2013) in Nordic Holsteins are not completely consistent with those of Fritz et al. (2013) for French Holsteins which are largely consistent with those in the summary of Cole et al. (2017) for US Holsteins. This would be expected if the majority of lethal mutations are quite recent in origin and if there is subdivision, possibly caused by different selection objectives in each of the subpopulations.

Cole et al. (2016) estimated that the economic losses due to reduced fertility and perinatal calf death in US Holsteins was almost US\$ 11 million per year and Charlier et al. (2016) estimated the cost due to embryonic losses from nine confirmed lethal loci to be NZ\$13.8 million in New Zealand dairy cattle and $€ 2.7$ million in Belgian Blue cattle. The use of available genotype data to detect and avoid carrier $\times$ carrier matings is clearly an effective way to improve the fertility of cattle, but may become difficult to implement as the number of detected lethal mutations and genetic defects increases within individual breeds. Cole (2015) has suggested an alternative approach in which the estimated genetic merits of individuals for net merit are adjusted for the economic losses due to fetal losses and has shown the method to be effective at reducing the frequency of recessive lethal alleles, whilst maintaining current rates of genetic improvement. Lethal alleles are also excellent candidates for multiplex genome editing in which the heterozygous deleterious alleles are simply removed from the genomes of the bulls that are placed into Al each generation (Hickey et al., 2016).

\section{Non-Mendelian effects on male fertility}

Loss-of-function lethal mutations behave in a manner that is called Mendelian inheritance because the ability of an allele to impact the viability of a progeny is the same if the allele is transmitted through the male or female germ lines. However, loci that are transmitted through the mitochondrial genome (or genomes in cases of heteroplasmy; about 5\% of individuals within mammalian species appear to have at least two mitochondrial genomes), on the $Y$ chromosome, or on the $\mathrm{X}$ chromosome can cause patterns of inheritance that are non-Mendelian. There are also cases of variants that are located on the non-sex associated autosomes that lead to phenotypes that are inherited in a non-Mendelian fashion. Imprinted loci are transmitted through the germ-line with either alleles transmitted by females being silenced (female imprinting in which case the male inherited allele is expressed) usually by DNA methylation or alleles transmitted by males being silenced (male imprinting). Each generation, the methylation status of these alleles is reset according to the sex of individual. Flisikowski et al. (2010) reported the segregation of a lethal locus in which the mode of inheritance appeared to be autosomal dominant with incomplete penetrance (not all individuals possessing the lethal allele die). Semen from a Finnish Ayrshire bull was used to artificially inseminate 1900 Finnish Ayrshire heifers and cows and field data collected by the Al Cooperative suggested that 
$42.6 \%$ of the late pregnancies attributed to the bull ended in stillbirths or abortions. This figure is close enough to a $50: 50$ segregation ratio to suggest that the bull was heterozygous for a mutation that was transmitted as a dominant lethal. The fact that lethality was slightly $<50 \%$ suggested that there could have been some progeny that inherited the lethal allele that did not die, presumably because they were protected by variants at other loci and this might explain why the bull itself escaped death. However, the causal variant was shown to be a $110 \mathrm{~kb}$ deletion within the MIMT1 gene, which is part of the PEG3 (paternally expressed gene) domain. As the alleles transmitted by males are expressed (the maternal alleles are silenced by methylation), both copies of MIMT1 were transmitted to progeny in activated form but progeny that inherited the deletion allele had no functional MIMT1 expression as all MIMT1 copies transmitted by their dams were silenced. The fact that the bull survived the inheritance of a lethal mutation was because he inherited the deletion MIMT1 allele in a silenced form from his dam and the functional MIMT1 allele was inherited from his sire. Thus, LOF mutations in MIMT1 have absolutely no effect on female fertility but are lethal when transmitted by the sire. MIMT1 encodes a non-coding RNA, which is not translated into a protein and has an unknown function. However, this naturally occurring mutation demonstrates that MIMT1 is essential to life. Magee et al. (2010) have also shown that SNPs within the PEG3 gene cluster are associated with calving, calf performance and fertility traits in Irish Holstein-Friesian cattle.

The bovine $Y$ chromosome comprises a small pseudoautosomal region with a homolog on the $\mathrm{X}$ chromosome and a much larger male-specific region which contains clusters of genes thought to be essential for male reproduction because they are primarily expressed during testicular development (Yang et al., 2011; Chang et al., 2013). The number of genes on the bovine $Y$ chromosome appears to be surprisingly large relative to the number of genes found on the human $Y$ chromosome suggesting a much greater potential role of the $Y$ chromosome in phenotype determination, particularly male fertility, in cattle than in humans (Chang et al., 2013). However, there appears to have been surprisingly little work conducted to date to characterize the effects of mutations in bovine $Y$ chromosome genes on male fertility. This is probably due to the fact that until recently, there was no reference sequence assembly for the bovine $Y$ chromosome and due to the highly repetitive and palindromic nature of the $Y$ chromosome sequence, the existing assembly is of much lower quality than the assembly for the autosomal genome. One study has shown that the number of copies of members of the HSFY and ZNF280BY gene families varies by almost an order of magnitude in cattle (from 20 to over 300 copies) and that variation in copy number of both families was negatively correlated with testis size, but positively correlated with SCR (Yue et al., 2014). Because the number of copies of members of both families within individual bulls is positively correlated (Yue et al., 2014), it is not clear which family (if either) was causal for effects on male fertility. Copy number variation in members of the PRAMEY gene family encoding proteins found in the sperm head and tail has been shown to be negatively correlated with percentage of normal sperm and non-return rate, but not with SCR in Holstein bulls (Yue et al., 2013).

Mutations within genes or regulatory regions on the $X$ chromosome have the potential to be severely deleterious to fertility in males who have only a single copy of the $X$ chromosome and far less so in females who have two copies of the $\mathrm{X}$ chromosome, although one copy is presumably randomly inactivated in each cell within every tissue. Because of this, and like $Y$ chromosome mutations, these variants are exposed to extremely strong purifying selection in males. Consequently, we might expect to find many fewer $X$ and $Y$ chromosome mutations affecting fertility than autosomal mutations. Despite this, using a GWAS in indicine and indicine $\times$ taurine bulls, Fortes et al. (2013b) found that the majority of genome-wide associations for scrotal circumference and percentage of normal spermatozoa at 24 months of age were located on the $X$ chromosome. De Camargo et al. (2015) examined the effects of seven SNPs responsible for amino acid substitutions in seven genes located in regions of the $X$ chromosome previously identified by the GWAS of Fortes et al. (2013b) in the same experimental population and detected significant associations for SNPs in LOC100138021, CENPI and TAF7L with percentage of normal spermatozoa (Table 2) and for SNPs in TEX11 and $A R$ with scrotal circumference. None of the SNPs detected as being associated with male fertility were found to be associated with female fertility. A brief overview of effects of chromosomal aberrations and structural variation on male fertility is presented in Supplementary Material S1.

\section{Quantitative trait loci responsible for variation in male fertility}

The evolution of DNA methylation probably occurred as a method to silence the transcriptional activity of retrotransposons that were integrated into the germ-line via retroviruses (Nagamori et al., 2015). DNA methylation appears to be ubiquitous in mammalian genomes; however, variation in the extent of DNA methylation at specific loci is known to occur and this variation has been associated with the quantitative regulation of gene expression. By examining the methylation profiles of DNA extracted from the spermatozoa of bulls with high- and low-conception rates, Verma et al. (2014) found differentially methylated regions associated with 151 genes with functions in germ cell development, spermatogenesis, capacitation, and embryonic development in water buffalo. Using a similar approach in Holstein bulls identified as being extreme for SCR based upon at least 300 inseminations, Kropp et al. (2017) found 76 genomic regions to be differentially methylated in the DNA extracted from the spermatozoa of high and low-SCR bulls. What is not clear from these epigenetic studies is whether the detected differential methylation is the cause of differences in fertility or 
Taylor, Schnabel and Sutovsky

Table 2 Genomic regions identified by genome-wide association analysis or candidate gene analysis as associated with sire conception rate

\begin{tabular}{|c|c|c|}
\hline SNP & Location (Chr: bp) & Gene(s) ${ }^{1}$ \\
\hline rs136195618 ${ }^{2}$ & $7: 41208950$ & PROP1 \\
\hline SNP11646 3 & $17: 35247641-35247490^{5}$ & FGF2 \\
\hline SNP12195 3,4 & $19: 43045658-43046477^{5}$ & STAT5A \\
\hline 1.5 Mb window ${ }^{6}$ & $5: 105357507-106813133$ & PARP11, AKAP3 \\
\hline$r s 42559373^{6,7}$ & $9: 11867269$ & RIMS1 \\
\hline 1.5 Mb window ${ }^{6}$ & $13: 58456868-59951247$ & CTCFL, SP011 \\
\hline rs42296108 & $15: 26472899$ & CADM1 \\
\hline $1.5 \mathrm{Mb}$ window ${ }^{6}$ & $21: 8031396-9528223$ & $I G F 1 R$ \\
\hline 1.5 Mb window ${ }^{6}$ & $21: 68846429-70294301$ & TDRD9, CKB \\
\hline rs110697318 & $21: 71210609$ & BRF1 \\
\hline $1.5 \mathrm{Mb}$ window ${ }^{6,7}$ & $25: 3148958-4647188$ & MGRN1, SEPT12 \\
\hline $1.5 \mathrm{Mb}$ window ${ }^{6}$ & $25: 26736589-28233820$ & CCT6A \\
\hline rs $41567516^{6}$ & $25: 27477941$ & KAT8 \\
\hline$r s 420651832^{6,8}$ & $25: 27672891$ & ITGAM \\
\hline rs416496816 & $25: 28711626$ & TYW1 \\
\hline rs $41642283^{9}$ & $2: 24837034$ & DYNC112 \\
\hline rs $109059438^{9}$ & $5: 112775479$ & LOC784935 \\
\hline rs $41571878^{9}$ & $18: 54965977$ & ZNF541 \\
\hline rs110629661, & $25: 983759$ & CACNA1H \\
\hline rs109758248 & $25: 2270518$ & LOC617302 \\
\hline rs110129888 & $25: 3898682$ & ROGDI \\
\hline rs110383224 & $25: 4229011$ & LOC521021 \\
\hline rs $42480223^{9}$ & $29: 14271174$ & - \\
\hline$r s 4125718^{10,11}$ & $1: 69802307$ & ITGB5 \\
\hline rs $2902486^{10,11}$ & $4: 11622140$ & COL1A2-AS1 \\
\hline rs29015574 & $9: 12042726$ & RIMS1 \\
\hline rs29016875 & $10: 6003240$ & SFNX1, DRD1 \\
\hline
\end{tabular}

SNP = single nucleotide polymorphism.

${ }^{1}$ SNP located within or near identified gene or window contains the listed genes. ${ }^{2}$ Lan et al. (2013).

${ }^{3}$ Estimated relative conception rate.

${ }^{4}$ Khatib et al. (2010)

${ }^{5}$ PCR amplicon contains identified SNP but exact reference genome position not provided.

${ }^{6}$ Han and Peñagaricano (2016).

${ }^{7}$ QTL validated in two or more studies.

${ }^{8}$ rs number corresponds to marker name but provided chromosomal coordinates are not correct.

${ }^{9}$ Peñagaricano et al. (2012).

${ }^{10}$ Validated in follow-up analysis of expanded genotyped sample.

${ }^{11}$ Feugang et al. (2009).

the effect of some other genomic mechanism that is responsible for the aberrant methylation of sequences regulating the expression of genes that are required for high fertility. Similarly, several studies have found differences in the spermatozoa mRNA transcript (Card et al., 2017), miRNA and piRNA (Capra et al., 2017) and protein (Peddinti et al., 2008) abundances. While these approaches have been pursued from the perspective of developing biomarkers of male fertility, the capability of the differentially abundant molecules to predict variation in male fertility has yet to be established.

There is an intrinsic relationship between GWAS studies and genomic selection. In GWAS, a large number of markers approximately evenly spread throughout the genome are assayed in a sample of phenotyped individuals and maker effects are individually tested to identify those that meet a pre-specified statistical threshold. This approach identifies the largest effect associations within the genome, based on the available sample size, and ignores the potential myriad of markers with small effects on the trait. These large effect markers can be used to generate molecular estimates of genetic merit, but typically these explain only small percentages of the overall additive genetic variance. In other words, there are relatively few markers of large effect and a much larger (but unknown) number of small effect markers. On the other hand, genomic selection attempts to use all of the markers (or a reasonably large subset of the markers when some Bayesian analyses are utilized) to predict genetic merit, capturing many more of the small effect variants, and can produce estimates of genetic merit that frequently explain at least $70 \%$ of the additive genetic variance in traits. In terms of application, it has historically been simpler and less expensive to genotype 10 or 20 markers in a large sample of individuals than many thousands of markers. However, with the recent deployment and very rapid adoption of highdensity SNP arrays for genotyping in cattle and the impact that genomic selection has had on the improvement of female fertility in US Holsteins (García-Ruiz et al., 2016), it is clear that the development of predictions of genetic merit for SCR should be a priority in cattle genomics. Two limitations to the approach are that all of the early industry genotyping was performed using the Illumina BovineSNP50 assay which did not include $Y$ chromosome variants (although all of the newer assays do), and the currently utilized statistical analyses do not appropriately model the effects of $X$ chromosome markers (Taylor, 2014). Imprinted loci are probably approximately correctly modelled in analyses of data from only a single sex, but are not correctly modelled in analyses of data from both sexes.

Feugang et al. (2009) estimated conception rates for 874 US Holstein bulls with an average of 788 breedings (range 101 to 11 997) in a probit analysis after adjusting for herd-year-month, parity, cow, days in milk and sire proven status. The 10 highest and 10 lowest fertility bulls (mean difference $15.4 \%$ in SCR) were genotyped using the Affymetrix/ParAllele 9919 SNP GeneChip and 8207 polymorphic markers were analysed in a single marker regression of allele dosage on each bull's SCR phenotype. The four most strongly associated SNPs $(P<0.0001$; Table 2$)$ were then genotyped in a larger cohort of 100 low- and 101 high-fertility bulls and the SNPs on chromosomes 1 and 4 were validated as being associated with SCR $(P<0.05)$. Peñagaricano et al. (2012) performed a GWAS in 1755 Holstein bulls with SCR data using 38650 SNPs with minor allele frequencies $>5 \%$ using a linear model correcting for relatedness among bulls and testing the effects of SNPs individually either fit with genotypes as additive, or additive and dominance effects. After correcting for multiple testing, they found eight SNPs defining five separate QTLs associated with SCR. Han and Peñagaricano (2016) performed a GWAS using 44449 estimated SCR animal effects (additive genetic + non-additive genetic and 
permanent environment effects; VanRaden P., USDA ARS, personal communication) available on 10884 US Holstein bulls. Of these animals, 7447 had high-density SNP genotype data and after filtering markers with minor allele frequencies $<1 \%$ or that were sex-linked, 58029 autosomal markers were analysed. A single-step BLUP analysis was used incorporating pedigree information for the animals that had not been genotyped. The percentage of additive genetic variance in SCR explained by all of the SNPs within $1.5 \mathrm{Mb}$ genomic regions was estimated and significant regions were declared when they explained at least $0.5 \%$ of the SCR additive genetic variance. The authors also performed a single-SNP analysis in which the mixed model included the effects described above but only for the genotyped animals and found results that were consistent with those from the single-step BLUP. Han and Peñagaricano (2016) estimated that the SNPs explained $32 \%$ of the variance in SCR animal effects and, consequently, $68 \%$ of the unexplained variance was due to additive genetic variance not captured by the SNPs, and the service sire non-additive genetic and permanent environmental effects. Han and Peñagaricano (2016) also found six SCR QTLs located on chromosomes 5, 13, 21 and 25 that explained at least $0.5 \%$ of the SCR additive genetic variance (Table 2).

As all three studies were conducted in US Holstein bulls, we should expect considerable consistency among the results and also for candidate gene studies previously reporting significant associations with SCR (Khatib et al., 2010; Lan et al., 2013). However, Table 2 shows that only two loci were consistently detected in at least two different studies in US Holsteins. Associations with RIMS1 on chromosome 9 between 11.8 and $12.1 \mathrm{Mb}$ were detected by Feugang et al. (2009) and Han and Peñagaricano (2016). Associations were also detected on chromosome 25 in the region from 0.9 to $4.7 \mathrm{Mb}$ by Peñagaricano et al. (2012) and Han and Peñagaricano (2016). This is somewhat typical of GWAS studies particularly when they are underpowered and there are a few variants of large effect responsible for trait variation. Druet et al. (2009) performed a GWAS with 148 microsatellite markers in 10 families containing 515 French Holstein bulls for semen production phenotypes of ejaculated volume and sperm concentration, number of spermatozoa, motility, velocity, percentage of motile spermatozoa after thawing and abnormal spermatozoa. Of the 11 detected QTL, only two affecting ejaculated volume (chromosome 15 at $22 \mathrm{cM}$ ) and sperm motility (chromosome 7 at $34 \mathrm{cM}$ ) appear to overlap with the SCR QTL reported in Table 2.

Abdollahi-Arpanahi et al. (2017) used the SCR data for 7447 bulls that was employed by Han and Peñagaricano (2016) for GWAS to evaluate the utility of genomic selection to predict the genetic merit of Al bulls for SCR using a fivefold cross-validation scheme and SNP feature selection. Using all 54706 fitted SNP, the average correlation between predicted genetic merit and SCR phenotype was 0.34 corresponding to a prediction accuracy of $\sim 0.63$. Selecting SNPs within genes with Gene Ontology and Medical Subject Heading terms including reproduction, fertilization, sperm motility or sperm capacitation did not improve prediction accuracy. However, restricting the analysis to the 18659 SNPs detected as being associated $\left(P_{\text {nominal }}<0.05\right)$ with SCR increased the correlation slightly to 0.35 . Nonlinear models uniformly outperformed linear models for accuracy of prediction, but the improvement was generally fairly small.

\section{Conclusions}

Male and female fertility are positively correlated but the correlation is low and genetic predictions for fertility are currently only produced for females. Genomic selection has produced dramatic increases in female fertility in a relatively short period of time in US Holsteins demonstrating that a low heritability is not the sole determinant of selection response. While this improvement should also have produced a small correlated response in male fertility, this is an unsatisfactory solution considering the economic importance of fertility to cattle production and the need to increase the efficiency and quantity of animal-based food proteins world-wide.

In dairy cattle, there is an opportunity to rapidly develop genomic predictions for male fertility (in both sexes) considering the large number of genotyped animals and the availability of SCR phenotypes. However, these phenotypes should be based on inseminations made by yearling bulls in which sperm dosages have been standardized and this will require collaboration between $\mathrm{Al}$ organizations. In the US beef industry, the majority of genetic improvement in all traits is created by selection within the registered sector. Despite the reduced use of Al relative to the dairy industry, it should similarly be possible to capture the benefits of increased rates of genotyping to develop genetic predictions for SCR. The increasing use of sexed semen to produce heifer replacements within the US dairy industry also presents an opportunity for the generation of SCR data for beef bulls, as sexed male semen from beef bulls is increasingly being used to breed dairy cows that were not selected to produce heifer replacements. Scoring conception rates in dairy cows is agnostic to the breed of bulls used in Al. Finally, increasing the rate of use of $\mathrm{Al}$ in commercial beef herds via the use of synchronization of oestrus and ovulation to facilitate fixed-time Al of beef cows has an enormous opportunity for the collection of SCR phenotypes in beef bulls. If $10 \%$ of the commercial beef cows in the US were bred by Al, the industry could collect more SCR phenotypes than are currently produced within the entire dairy industry. If genomic predictions of merit for male fertility are to be produced for both males and females, efforts should be invested to develop and evaluate models that appropriately model the effects of sex chromosome and imprinted variants.

\section{Acknowledgements}

The authors appreciate the support of NIH-USDA Dual Purpose with Dual Benefit Program grant number NIH 1R01HD084353. PS is also supported by USDA-NIFA grant 2015-67015-23231 
and by seed funding from the Food for the 21st Century Program of the University of Missouri. J.T. and R.S. are supported by USDA-NIFA grants 2013-68004-20364, 2016-67015-24923 and 2017-67015-26760. The authors also appreciate the constructive criticisms of two anonymous reviewers who appreciably improved the manuscript.

\section{Declaration of interest}

The authors have no conflicts of interest.

\section{Ethics statement}

No animals were used in this review study.

\section{Software and data repository resources}

No software or unpublished data were used in this review.

\section{Supplementary material}

To view supplementary material for this article, please visit https://doi.org/10.1017/S1751731118000599

\section{References}

Abdollahi-Arpanahi R, Morota G and Peñagaricano F 2017. Predicting bull fertility using genomic data and biological information. Journal of Dairy Science 100, 9656-9666.

Berry DP, Wall E and Pryce JE 2014. Genetics and genomics of reproductive performance in dairy and beef cattle. Animal 8 (suppl. 1), 105-121.

Capra E, Turri F, Lazzari B, Cremonesi P, Gliozzi TM, Fojadelli I, Stella A and Pizzi $F$ 2017. Small RNA sequencing of cryopreserved semen from single bull revealed altered miRNAs and piRNAs expression between high- and low-motile sperm populations. BMC Genomics 18, 14.

Card CJ, Krieger KE, Kaproth M and Sartini BL 2017. Oligo-dT selected spermatozoal transcript profiles differ among higher and lower fertility dairy sires. Animal Reproduction Science 177, 105-123.

Chang TC, Yang Y, Retzel EF and Liu WS 2013. Male-specific region of the bovine $Y$ chromosome is gene rich with a high transcriptomic activity in testis development. Proceedings of the National Academy of Science USA 110, 12373-12378.

Charlier C, Li W, Harland C, Littlejohn M, Coppieters W, Creagh F, Davis S, Druet T, Faux P, Guillaume F, Karim L, Keehan M, Kadri NK, Tamma N, Spelman R and Georges M 2016. NGS-based reverse genetic screen for common embryonic lethal mutations compromising fertility in livestock. Genome Research 26, 1333-1341.

Chen W-H, Lu G, Chen X, Zhao X-M and Bork P 2017. OGEE v2: an update of the online gene essentiality database with special focus on differentially essential genes in human cancer cell lines. Nucleic Acids Research 45, D940-D944.

Cole JB 2015. A simple strategy for managing many recessive disorders in a dairy cattle breeding program. Genetics Selection Evolution 47, 94.

Cole JB, Null DJ and VanRaden PM 2016. Phenotypic and genetic effects of recessive haplotypes on yield, longevity, and fertility. Journal of Dairy Science 99, 7274-7288.

Cole JB, VanRaden PM, Null DJ, Hutchison JL, Cooper TA and Hubbard SM 2017. Haplotype tests for recessive disorders that affect fertility and other traits. Retrieved on 30 November 2017 from https://aipl.arsusda.gov/reference/recessive_haplotypes_ARR-G3.html.

David I, Bodin L, Lagriffoul G, Leymarie C, Manfredi E and Robert-Granié C 2007. Genetic analysis of male and female fertility after artificial insemination in sheep: comparison of single-trait and joint models. Journal of Dairy Science 90 , 3917-3923.

De Camargo GM, Porto-Neto LR, Kelly MJ, Bunch RJ, McWilliam SM, Tonhati H, Lehnert SA, Fortes MR and Moore SS 2015. Non-synonymous mutations mapped to chromosome $\mathrm{X}$ associated with andrological and growth traits in beef cattle. BMC Genomics 16, 384.
DeJarnette JM, Nebel RL and Marshall CE 2010. Understanding estimates of Al sire fertility. Retrieved on 18 December 2017 from http://www.selectsires.com/ programs/docs/UnderstandingAISireFertility.pdf?version $=20170404$.

Druet T, Fritz S, Sellem E, Basso B, Gérard O, Salas-Cortes L, Humblot P, Druart X and Eggen A 2009. Estimation of genetic parameters and genome scan for 15 semen characteristics traits of Holstein bulls. Journal of Animal Breeding and Genetics 126, 269-277.

Feugang JM, Kaya A, Page GP, Chen L, Mehta T, Hirani K, Nazareth L, Topper E, Gibbs R and Memili E 2009. Two-stage genome-wide association study identifies integrin beta 5 as having potential role in bull fertility. BMC Genomics 10,176 .

Flisikowski K, Venhoranta H, Nowacka-Woszuk J, McKay SD, Flyckt A, Taponen J, Schnabel R, Schwarzenbacher H, Szczerbal I, Lohi H, Fries R, Taylor JF, Switonski M and Andersson M 2010. A novel mutation in the maternally imprinted PEG3 domain results in a loss of MIMT1 expression and causes abortions and stillbirths in cattle (Bos taurus). PLoS One 5, e15116.

Fortes MRS, DeAtley KL, Lehnert SA, Burns BM, Reverter A, Hawken RJ, Boe-Hansen G, Moore SS and Thomas MG 2013a. Genomic regions associated with fertility traits in male and female cattle: advances from microsatellites to high-density chips and beyond. Animal Reproduction Science 141, 1-19.

Fortes MR, Reverter A, Kelly M, McCulloch R and Lehnert SA 2013b. Genome-wide association study for inhibin, luteinizing hormone, insulin-like growth factor 1, testicular size and semen traits in bovine species. Andrology 1, 644-650.

Fritz S, Capitan A, Djari A, Rodriguez SC, Barbat A, Baur A, Grohs C, Weiss B, Boussaha M, Esquerre D, Klopp C, Rocha D and Boichard D 2013. Detection of haplotypes associated with prenatal death in dairy cattle and identification of deleterious mutations in GART, SHBG and SLC37A2. PLoS One 8, e65550.

García-Ruiz A, Cole JB, VanRaden PM, Wiggans GR, Ruiz-López FJ and Van Tassell CP 2016. Changes in genetic selection differentials and generation intervals in US Holstein dairy cattle as a result of genomic selection. Proceedings of the National Academy of Sciences USA 113, E3995-E4004.

Han Y and Peñagaricano F 2016. Unravelling the genomic architecture of bull fertility in Holstein cattle. BMC Genetics 17, 143.

Hansen M 1979. Genetic investigations on male and female fertility in cattle. Livestock Science 6, 325-334.

Hickey JM, Bruce C, Whitelaw A and Gorjanc G 2016. Promotion of alleles by genome editing in livestock breeding programmes. Journal of Animal Breeding and Genetics 133, 83-84.

Hoff JL, Decker JE, Schnabel RD and Taylor JF 2017. Candidate lethal haplotypes and causal mutations in Angus cattle. BMC Genomics 18, 799.

Kadri NK, Sahana G, Charlier C, Iso-Touru T, Guldbrandtsen B, Karim L, Nielsen US, Panitz F, Aamand GP, Schulman N, Georges M, Vilkki J, Lund MS and Druet T 2014. A 660-Kb deletion with antagonistic effects on fertility and milk production segregates at high frequency in Nordic Red cattle: additional evidence for the common occurrence of balancing selection in livestock. PLoS Genetics 10, e1004049.

Khatib H, Monson RL, Huang W, Khatib R, Schutzkus V, Khateeb H and Parrish JJ 2010. Short communication: validation of in vitro fertility genes in a Holstein bull population. Journal of Dairy Science 93, 2244-2249.

Kropp J, Carrillo JA, Namous H, Daniels A, Salih SM, Song J and Khatib H 2017. Male fertility status is associated with DNA methylation signatures in sperm and transcriptomic profiles of bovine preimplantation embryos. BMC Genomics $18,280$.

Lan XY, Peñagaricano F, DeJung L, Weigel KA and Khatib H 2013. Short communication: a missense mutation in the PROP1 (prophet of Pit 1) gene affects male fertility and milk production traits in the US Holstein population. Journal of Dairy Science 96, 1255-1257.

Magee DA, Sikora KM, Berkowicz EW, Berry DP, Howard DJ, Mullen MP, Evans RD, Spillane $C$ and MacHugh DE 2010. DNA sequence polymorphisms in a panel of eight candidate bovine imprinted genes and their association with performance traits in Irish Holstein-Friesian cattle. BMC Genetics 11, 93.

Matukumalli LK, Lawley CT, Schnabel RD, Taylor JF, Allan MF, Heaton MP, O'Connell J, Moore SS, Smith TPL, Sonstegard TS and Van Tassell CP 2009. Development and characterization of a high density SNP genotyping assay for cattle. PLoS One 4, e5350.

Mesbah-Uddin M, Guldbrandtsen B, Iso-Touru T, Vilkki J, De Koning DJ, Boichard D, Lund MS and Sahana G 2018. Genome-wide mapping of large deletions and their population-genetic properties in dairy cattle. DNA Research 25, 49-59. 
Meuwissen TH, Hayes BJ and Goddard ME 2001. Prediction of total genetic value using genome-wide dense marker maps. Genetics 157, 1819-1829.

Meyer K, Hammond K, Mackinnon MJ and Parnell PF 1991. Estimates of covariances between reproduction and growth in Australian beef cattle. Journal of Animal Science 69, 3533-3543.

Nagamori I, Kobayashi H, Shiromoto Y, Nishimura T, Kuramochi-Miyagawa S, Kono T and Nakano T 2015. Comprehensive DNA methylation analysis of retrotransposons in male germ cells. Cell Reports 12, 1541-1547.

Pausch H, Schwarzenbacher H, Burgstaller J, Flisikowski K, Wurmser C, Jansen S, Jung S, Schnieke A, Wittek T and Fries R 2015. Homozygous haplotype deficiency reveals deleterious mutations compromising reproductive and rearing success in cattle. BMC Genomics 16, 312-325.

Peddinti D, Nanduri B, Kaya A, Feugang JM, Burgess SC and Memili E 2008. Comprehensive proteomic analysis of bovine spermatozoa of varying fertility rates and identification of biomarkers associated with fertility. BMC Systems Biology 2, 19.

Peñagaricano F, Weigel KA and Khatib H 2012. Genome-wide association study identifies candidate markers for bull fertility in Holstein dairy cattle. Animal Genetics 43 (suppl. 1), 65-71.

Piles $M$ and Tusell L 2012. Genetic correlation between growth and female and male contributions to fertility in rabbit. Journal of Animal Breeding and Genetics 129, 298-305.

Sahana G, Nielsen US, Aamand GP, Lund MS and Guldbrandtsen B 2013 Novel harmful recessive haplotypes identified for fertility traits in Nordic Holstein cattle. PLoS One 8, e82909.

Sahana G, Iso-Touru T, Wu X, Nielsen US, de Koning DJ, Lund MS, Vilkki J and Guldbrandtsen B 2016. A $0.5-\mathrm{Mbp}$ deletion on bovine chromosome 23 is a strong candidate for stillbirth in Nordic Red cattle. Genetics Selection Evolution $48,35$.

Syrstad 0 1981. Selection for fertility on the basis of Al data. Livestock Production Science 8, 247-252.

Taylor JF 2014. Implementation and accuracy of genomic selection. Aquaculture 420-421, S8-S14.

Taylor JF, Schnabel RD and Sutovsky P 2018. Identification of genomic variants causing sperm abnormalities and reduced male fertility. Animal Reproduction Science, pii: S0378-4320(17)31072-2, https://doi.org/10.1016/j.anireprosci.2018. 02.007, Published online 10 February 2018.

VanRaden PM, Olson KM, Null DJ and Hutchison JL 2011. Harmful recessive effects on fertility detected by absence of homozygous haplotypes. Journal of Dairy Science 94, 6153-6161.

Verma A, Rajput S, De S, Kumar R, Chakravarty AK and Datta TK 2014. Genome-wide profiling of sperm DNA methylation in relation to buffalo (Bubalus bubalis) bull fertility. Theriogenology 82, 750-759.

Wolc A, White IM, Olori VE and Hill WG 2009. Inheritance of fertility in broiler chickens. Genetics Selection Evolution 41, 47.

Yang Y, Chang TC, Yasue H, Bharti AK, Retzel EF and Liu WS 2011. ZNF280BY and ZNF280AY: autosome derived Y-chromosome gene families in Bovidae. BMC Genomics 12, 13.

Yue XP, Chang TC, DeJarnette JM, Marshall CE, Lei CZ and Liu WS 2013. Copy number variation of PRAMEY across breeds and its association with male fertility in Holstein sires. Journal of Dairy Science 96, 8024-8034.

Yue XP, Dechow C, Chang TC, DeJarnette JM, Marshall CE, Lei CZ and Liu WS 2014. Copy number variations of the extensively amplified Y-linked genes, HSFY and ZNF280BY, in cattle and their association with male reproductive traits in Holstein bulls. BMC Genomics 15, 113. 